\title{
A Virtual EMG Signal Control and Analysis for Optimal Hardware Design
}

\author{
https://doi.org/10.3991/ijoe.v18i02.27047
}

Hadeel K. Aljobouri

College of Engineering, Al-Nahrain University, Baghdad, Iraq

hadeel_bme77@yahoo.com

\begin{abstract}
A muscle-computer interface is one of the new applications of the human-computer interface technologies and specifically the brain-computer interface. Brain-muscle-computer interface based on the Electromyography (EMG) signal. EMG signal is an electrical activity from a muscle that is used as an input for effecting several tasks. This work presented an interfacing process between the Graphical User Interface (GUI) and hardware system. Using the implemented system, the researcher shall deals with the raw EMG data easily by analyzing the signal from the muscle sensor detection. A novel virtual EMG signal control and analysis system design is proposed in this work. The system consists mainly of two parts, hardware and software toolbox. Hardware design is mainly dependent on using a muscle movement sensor as well as the feedback from the virtual toolbox. The virtual software design offers a relatively simple design of a friendly graphical user interface. It consists mainly of the input EMG signal and output signal after using different processing methods. Feedback response from the final EMG signal results after the processing may help the designer to present the optimal hardware design. The output results show the output performance of the proposed virtual EMG data controlling and analysis with the implemented hardware design of the muscle sensor movement detection. The results show promise that these interfaces may provide a new option to benefit the designer in choosing the optimal prosthesis design of severely disabled persons.
\end{abstract}

Keywords - paper publishing, biomedical signal analysis, EMG sensors, GUI, human-computer interface

\section{$1 \quad$ Introduction}

Electromyography (EMG) signals have been effectively used in different medical fields like medical cases [1], [2], Control of the prosthetic arm with force estimation [3], [4], and fatigue detection with different methods [5]-[7]. There is many information presented on voluntaries that is related to muscle-computer interface such as EMG and muscle signal processing [8]-[17], brain-computer interface [18], [19] and etc.

The Brain-muscle-computer interface is one of the helpful applications of the human-computer interface technologies to overcome many human limitations. EMG 
signal is an electrical activity from the muscle that is used as an input for effecting several tasks, such as pain management, gait analysis, orthotics, prosthetic device control, and human-computer interfaces [20]-[22].

E. Clancy in 2003, presented a new circuit design for EMG signal detection and conditioning. The performance characteristics of the proposed design were satisfied, but the power consumption of the circuit was high with respect to the new technologies. Based on Clancy's circuit design, M. Mozhanova improved the design regarding power consumption, size, and performance [23].

Zecca et al. presented a simplified graph for a multifunctional prosthesis hand and how it could be controlled by the EMG signal [24]. The introduced mechatronic device consists of signal acquisition and processing, actuators, sensors, control, and batteries. They illustrated that the main restriction of controlling the artificial device would continue even when the number of DoFs of the prosthesis could be increased. Therefore, the prosthetic user interface must be suitable to enable the subject for practical long-term use of the prosthesis with spending low energy.

In 2019, the development of a low-cost prosthetic hand was introduced by Budiharto [25]. The presented device is based on using a 3-lead EMG sensor combined with 1 channel electroencephalograph (EEG). The initial output results were satisfied using EMG and EEG signal but still required improvement for using the EEG signal.

Regarding the brain-muscle computer interface, Joshi et al. advanced a different human-computer interface using a single surface EMG signal [26]. They formed a new brain-muscle-computer interface that can be used in simple two-dimensions cursor-to-target tasks. They trained several subjects with complex visual feedback control of external devices. The output results presented the subjects' ability to hit targets on a computer screen with a cursor.

In 2016, Landa-Jiménez et al. introduced a low-cost and simple brain-musclecomputer interface [27]. The presented design is constructed as an educational tool for students in the field of neuroscience, physiology, and bioengineering courses. This design can describe theoretical and practical applications. As well as the explanation about the EMG signals and physiology of the muscle and motor unit, the students could learn how the system controlled a robot using surface EMG signal. Muscle computer interface constructed from the Backyard Brains EMG SpikerBox bioamplfier, Arduino board, and Bluetooth module. Using EMG muscle contractions, the designed system can control a LEGO robot.

A new friendly virtual EMG signal control and analysis interfacing design are presented in this work. The proposed system consists mainly of two parts, hardware and software toolbox. Hardware design is depended on the muscle contractions as well as the feedback from the virtual toolbox. The virtual software design offers a relatively simple design of graphical user interfaces.

The rest of this article is organized as follows. The virtual design and hardware construction are described in Section 2. All the experimental output results for the Hardware Architecture design and EMG Visualization and Analysis system are presented in Sections 3 and 4 respectively. Finally, the discussion and conclusions are presented in Sections 5 and 6, respectively. 


\section{Hardware construction and virtual design}

Many brain-muscle computer interfaces have been developed for measuring the muscle's signals based on the brain's ability to learn neuromuscular abilities via operant conditioning [28] [29]. The main interfacing system presented in this work consists of the friendly Graphical User Interface (GUI) design and hardware system. The EMG signal is recorded when the muscle fibers of the residual prosthesis are excited. Then the obtained signal is fed to the servomotors and the GUI window to be processed. The designer or researcher can use the facilities of the visual feedback for controlling and choosing an optimal design before implementing the final one.

This process is presented in a simplified block diagram shown in Figure 1. The proposed system is divide mainly into hardware and virtual design. Each part is explained in detail in the following sections.
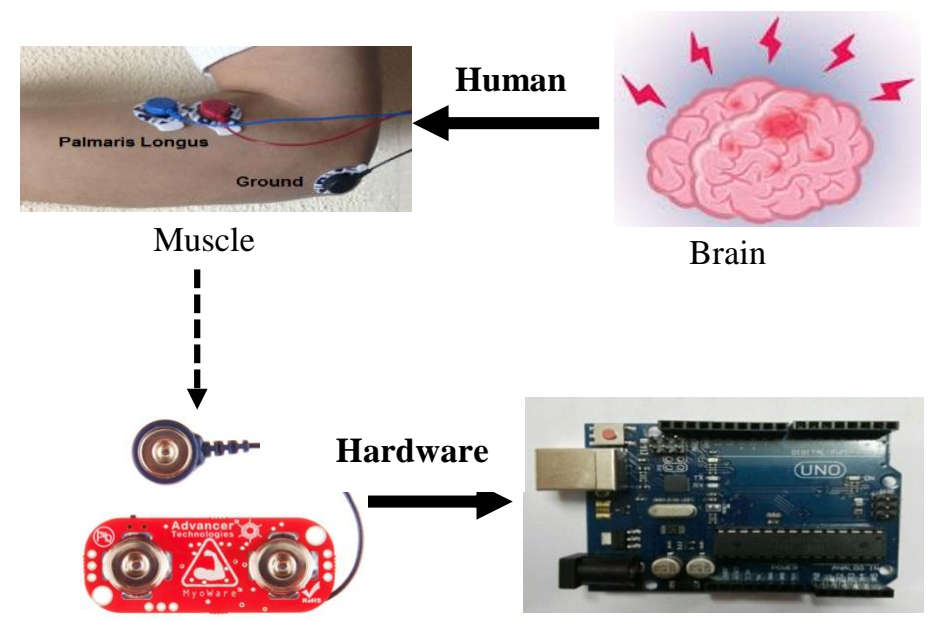

Mayware sensor

Microcontroller

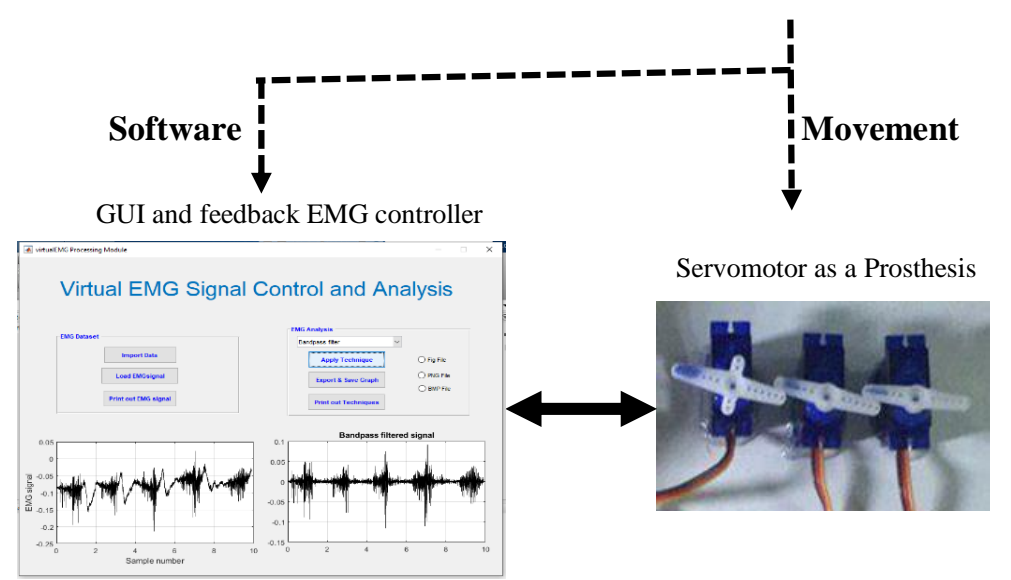

Fig. 1. A simplified block diagram of the proposed system 


\section{Hardware architecture and experimental results}

The hardware system is implemented for controlling the motor movement as a virtual representation of the prosthetic movement. The implemented muscle-computer interface design is shown in Figure 2. It is consists of the electrodes, Myoware Muscle sensor, Arduino board, servo motors, and PC. Each component of the basic hardware system is controllable with the aids of the EMG software package which has explained in detail in the next section.

The EMG sensor adheres to the skin through the bipolar $\mathrm{Ag} / \mathrm{AgCl}$ surface electrodes. These electrodes are simply made of silver or platinum [30]. The Myoware Muscle sensor is an EMG sensor [31]. This type of sensor is powered with Arduino and accessible in the markets. It is used for measuring filtered and rectified electrical muscle activity through the process of differential amplification. Myoware sensor has three electrodes that are put directly in the skin with no cables to reduce the artifacts that occur during the use of the standard sensors that have wires. These electrodes are arranged to work as a differential amplifier. Two electrodes were used for recording the energy from muscle and one reference electrode.

Arduino board [32] is used as a microcontroller, which is a programmable controller (Arduino Uno). It is programmed by a computer and runs using Arduino (IDE) software that controls all the hardware components. Arduino Nano has been used because of its small size and lightweight, so it is suitable for a prosthetic hand.

Servo motors arranged for an accurate control concerning velocity and angular position that is used as an actuator [33]. The three wires of each one of the three servo motors have connected to Arduino through the VIN, Ground, and D3, D5, and D6, which is connected to the Pulse-Width Modulation (PWM) wire of each motor. In our work, the electrodes and sensors are placed on the flexor carpi ulnaris muscle. The Three servo motors can provide an excellent and accurate movement for a prosthetic hand.

When the hand has closed, the muscle contracted, and the EMG signal will be one, so the microcontroller sends the command to the servo motors to move at angle ninety (Figure 3). While when the hand has opened, the muscle relaxed, and the EMG signal will be zero, so the microcontroller sends the command to the servo motors to move at angle zero (Figure 4). 


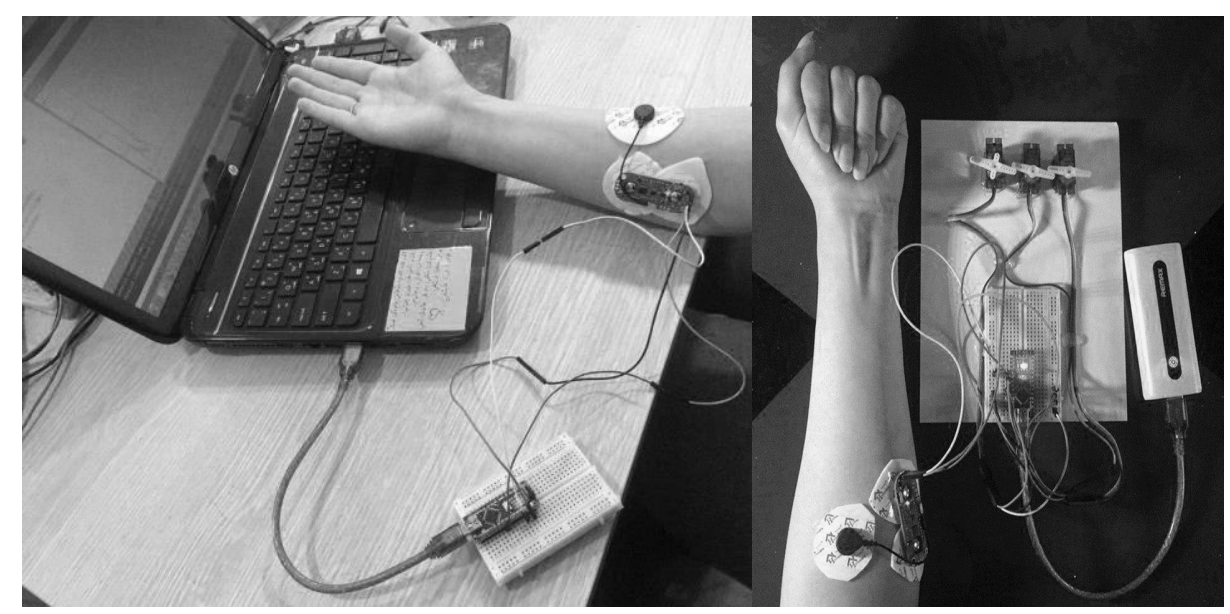

Fig. 2. The implemented muscle-computer interface design. A: Myoware Muscle sensor. B: ground electrode. C: electrode 1. D: electrode 2. E: Arduino board. F: servo motors. G: PC

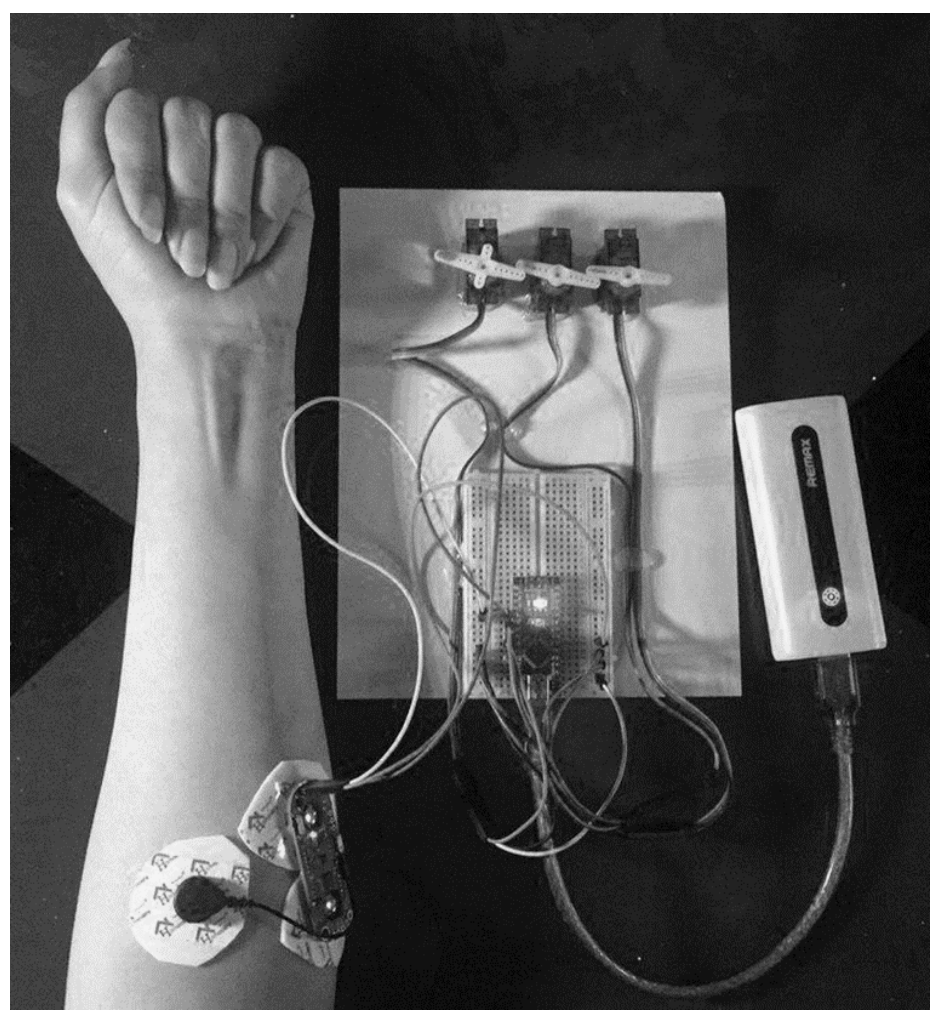

Fig. 3. The hand is closed 


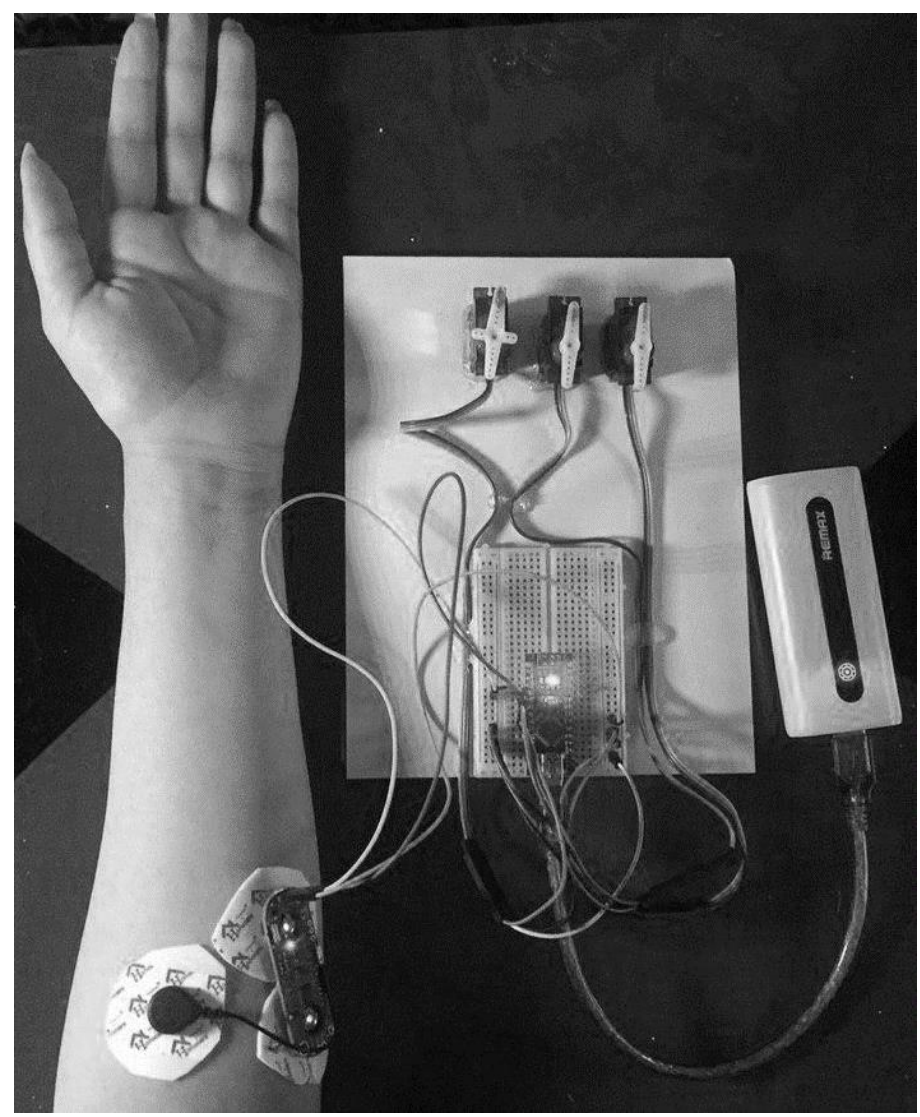

Fig. 4. The hand is opened

\section{EMG visualization and analysis: Experimental results}

The purpose of this section is to present the EMG visualization GUI tool after acquiring the EMG signal from the hardware system. The user-friendly toolbox was designed based on the MATLAB R2017b platform. The flow chart in Figure 5 simply summarizes the usage of the designed GUI tool. The designed tool consists of two main parts, the "EMG Dataset" and the "EMG Analysis" part. The first part is the "EMG Dataset" part, and the data import and selection process are executed in this part of the tool. At the start of the application, the first step is to import the EMG dataset from a data file where the EMG signal is stored after the Arduino process. When the user clicks the "Import Data" button, the browsing window in Figure 6 appears. The user can browse the files and select single dataset files (such as .txt, .mat, etc.) using this window. After a file selection process, the EMG dataset is loaded and appeared in the left window as shown in Figure 7. The user can print the output signal through clicks the "Print out EMG signal" button as shown in Figure 8. 
The second part of the designed tool is the "EMG Analysis" module which consists of a Bandpass filter, Rectifier filter, and Normalization. For the selected data in the first part, processing with the selected filter has been performed. The output results are shown in the right window as shown in Figure 7.

The EMG raw data is filtered with a second-order band-pass Butterworth filter between $20 \mathrm{~Hz}$ and $500 \mathrm{~Hz}$ frequencies. The sampling frequency of the filter is equal to the sampling frequency of the signal. The filter was used to clean up the signal and remove the artifacts. There are different sources of the artifacts which depend on many elements that affect signal quality, such as:

- common-mode rejection ratio (CMRR) and amplifier specification

- the feature of the surface electrodes

- the feature of the cables used, if they are shielded or unshielded

- Hand movement through the recording

Then, the EMG data is rectified to a full-wave to take the absolute value of the waveform and convert the input signal to one of constant polarity, either negative or positive, at its output.

Finally, the EMG data is normalized to its maximum value between 0 and 1 . Normalizing the waveform is necessary to compare the resultant waveform to a reference one that may not have the same range of voltage. The output EMG signal can be exported and saved as .Fig, .PNG, or .BMP. The user can print the output signal after applying the analysis technique through clicks the "Print out Techniques" button.

The proposed GUI system includes various components to help record and processing the EMG signal, train the user, and control various devices. This system is developed for Controlling the Prosthetic by Processing the EMG Signal. 


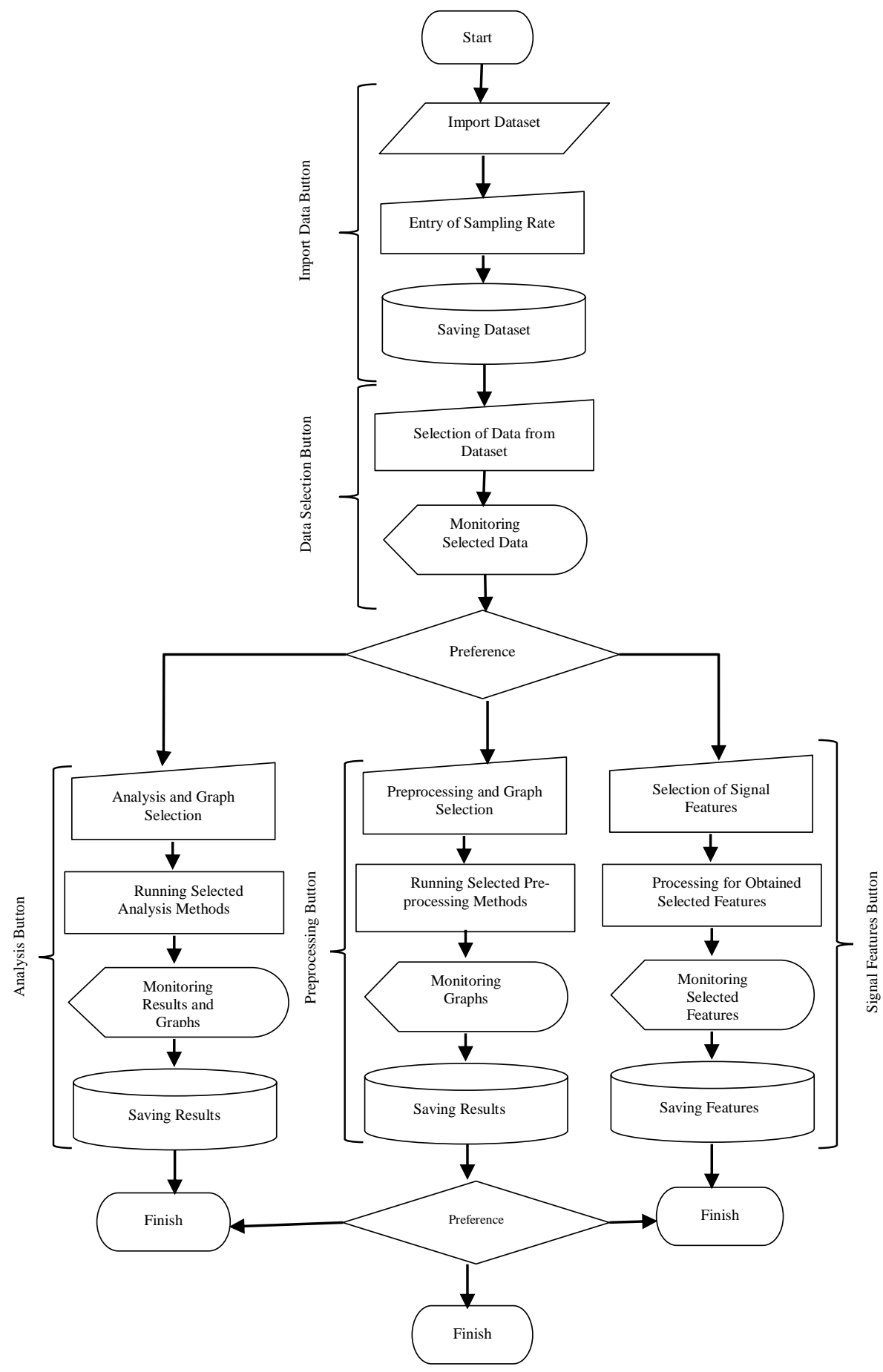

Fig. 5. The flowchart of the designed tool 


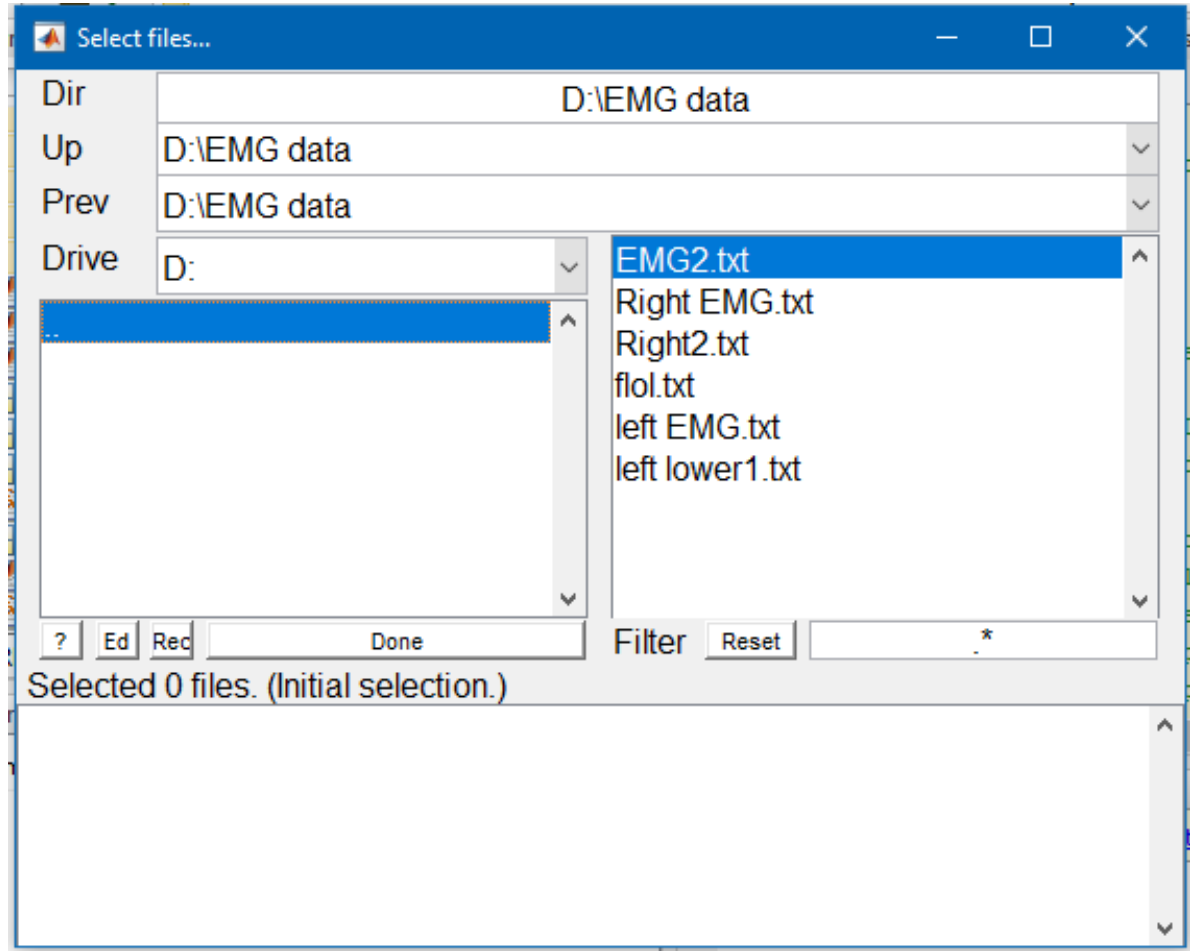

Fig. 6. Selection of a data file

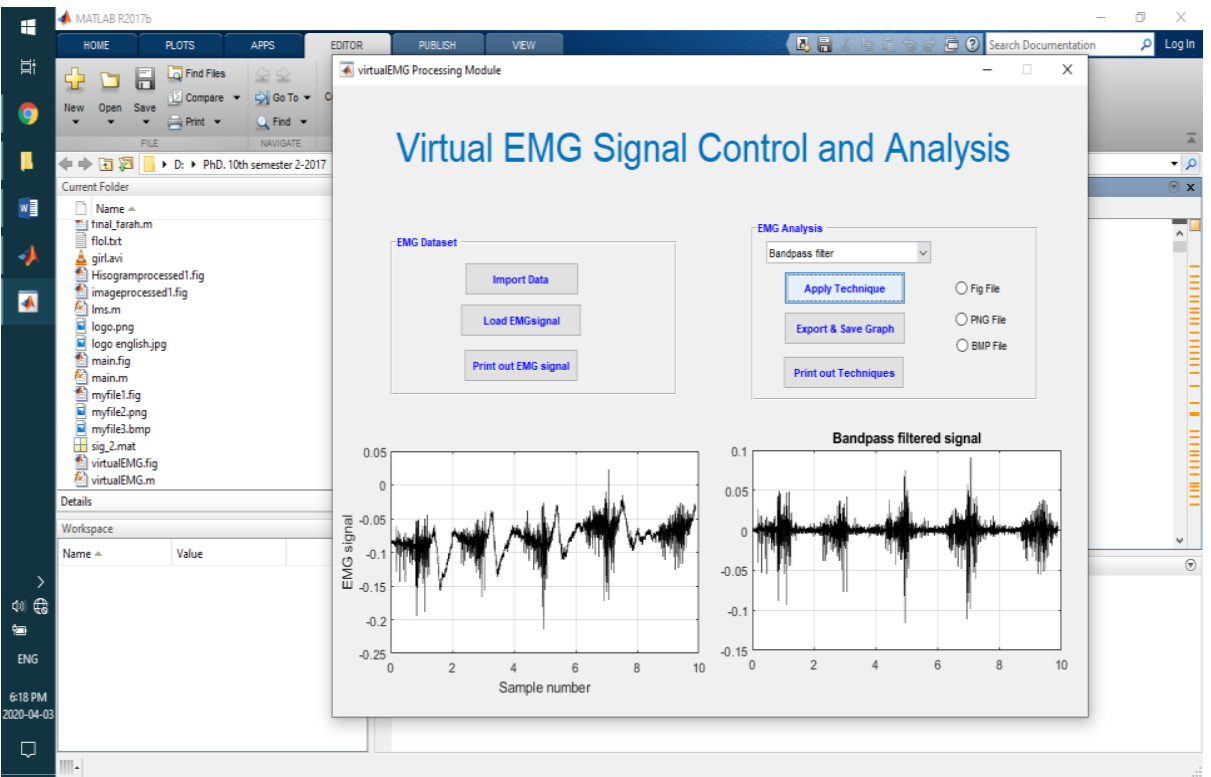

Fig. 7. Formal scheme for acquisition and analysis of EMG for control of prosthetic devices 


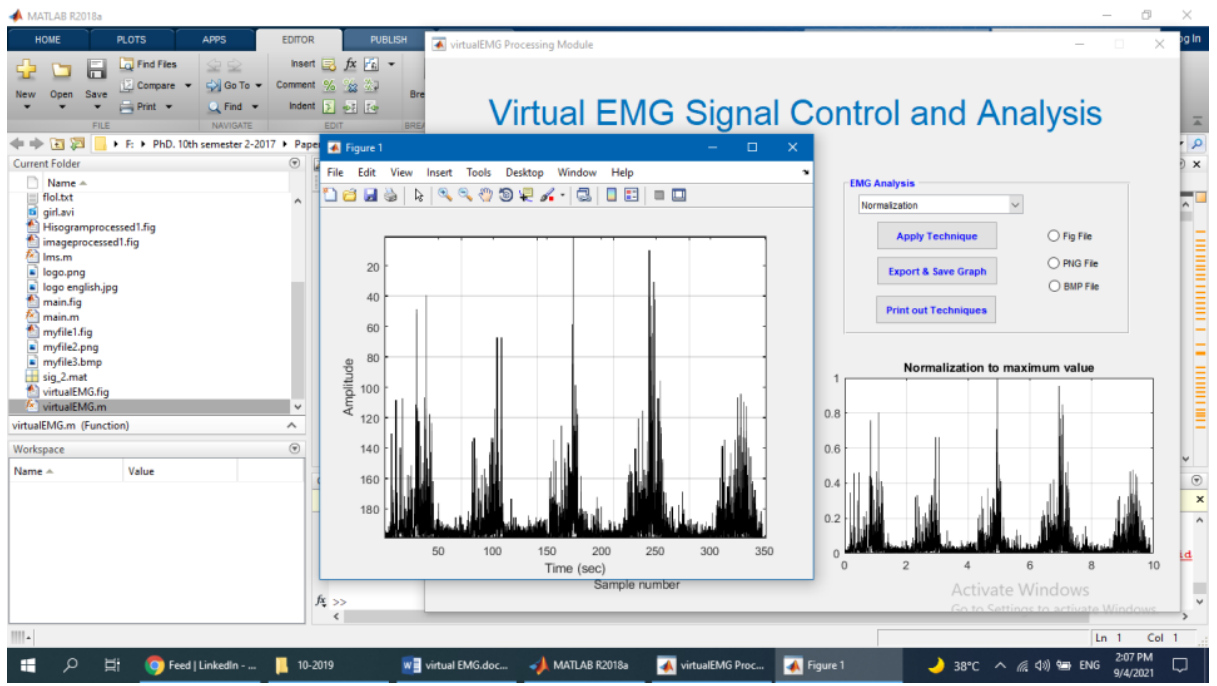

Fig. 8. Print the output technique (Normalization as an example)

\section{Discussion}

The procedure described above is followed to extract the simulated signals available through muscle contraction and relaxation to the virtual toolbox. The Feedback response from the final EMG signal results after the processing can support the designer to present the optimal hardware design. The collection between the hardware system and the virtual tool gives a novelty concerning the traditional projects [23], [24], [26], [27]. The EMG dataset extracted is available for clinical and healthcare clarification.

The presented work is of vital importance for the medical and biomedical equipment lab, so the student can be familiar with the working of this type of important artificial medical device. The researcher can deal with the device and record different readings. This work is simple and not costly.

\section{Conclusion}

One of the basic ideas of the designed GUI software package is to provide a virtual EMG control multifunctional prosthetic system. The presented package has the capability of simulating simple EMG recordings and analysis but without the complexity of applying the digital signal processing techniques, only deals with few buttons. The final results give feedback for improving the proposed hardware design for working in a good performance and robust form.

The presented work introduced an interfacing process between the GUI and hardware design. The researcher using the implemented system shall deals with the raw 
EMG data easily. Specifically, a researcher who has successfully met the objectives will be able to:

- Explain the interaction between the GUI and the implemented hardware system design.

- Describe the major components of the EMG muscle sensor hardware system and GUI window.

- Identify what is needed to obtain a final EMG signal.

- Identify which filters are relevant for obtaining a good signal as well as what is the output after using each a specified filter.

- Identify the ranges of typical values of these parameters.

- Explain how these signals are related and their effects on motor movement.

In general, the user will be able to analyze the raw signal from the muscle sensor detection and give feedback for the motor that represents the prosthesis movement.

The main objective of the proposed work is to introduce a new muscle-computer interfacing system. The presented system will be used by the physician and healthcare staff to find an optimal prosthetic design before implementation. Therefore, they can overcome many human prosthesis limitations such as repairing the robotic prosthetic response before implementing the final design.

In the future, the hardware design can be attached to a 3D printed prosthetic hand to construct a complement system. Also, use more EMG muscle sensors as a channel, use five motors instead of three for more control of movement and also perform the programming to be more efficient and to simulating more gestures.

\section{$7 \quad$ References}

[1] M. A. Crary, G. D. Carnaby, M. E. Groher, and E. Helseth, "Functional benefits of dysphagia therapy using adjunctive sEMG biofeedback," Dysphagia, vol. 19, no. 3, pp. 160-164, 2004. https://doi.org/10.1007/s00455-004-0003-8

[2] C. Disselhorst-Klug, T. Schmitz-Rode, and G. Rau, "Surface electromyography and muscle force: Limits in sEMG-force relationship and new approaches for applications," Clinical Biomechanics, vol. 24, no. 3. Elsevier, pp. 225-235, Mar. 01, 2009. https://doi. org/10.1016/j.clinbiomech.2008.08.003

[3] E. Park and S. G. Meek, "Adaptive Filtering of the Electromyographic Signal for Prosthetic Control and Force Estimation," IEEE Transactions on Biomedical Engineering, vol. 42, no. 10, pp. 1048-1052, 1995. https://doi.org/10.1109/10.464381

[4] E. Yulianto, T. B. Indrato, B. T. M. Nugraha, and S. Suharyati, "Wheelchair for Quadriplegic Patient with Electromyography Signal Control Wireless," International Journal of Online and Biomedical Engineering (iJOE), vol. 16, no. 12, pp. 94-115, Oct. 2020, Accessed: Sep. 21, 2021. https://doi.org/10.3991/ijoe.v16i12.15721

[5] M. González-Izal, A. Malanda, E. Gorostiaga, and M. Izquierdo, "Electromyographic models to assess muscle fatigue," Journal of Electromyography and Kinesiology, vol. 22, no. 4. pp. 501-512, Aug. 2012. https://doi.org/10.1016/j.jelekin.2012.02.019

[6] I. Cosic, S. L. Giudice, J. Hawley, D. K. Kumar, and V. P. Singh, "Strategies to identify changes in SEMG due to muscle fatigue during cycling.," Conference proceedings : ... Annual International Conference of the IEEE Engineering in Medicine and Biology 
Society. IEEE Engineering in Medicine and Biology Society. Annual Conference, vol. 2005, pp. 6683-6. https://doi.org/10.1109/iembs.2005.1616036

[7] A. Subasi and M. K. Kiymik, "Muscle fatigue detection in EMG using time-frequency methods, ICA and neural networks," Journal of Medical Systems, vol. 34, no. 4, pp. 777785, Aug. 2010. https://doi.org/10.1007/s10916-009-9292-7

[8] F. Q. Xiong and E. Shwedyk, "Some Aspects of Nonstationary Myoelectric Signal Processing," IEEE Transactions on Biomedical Engineering, vol. BME-34, no. 2, pp. 166172, 1987. https://doi.org/10.1109/TBME.1987.326041

[9] P. A. O’Neill, E. L. Morin, and R. N. Scott, "Myoelectric Signal Characteristics from Muscles in Residual Upper Limbs," IEEE Transactions on Rehabilitation Engineering, vol. 2, no. 4, pp. 266-270, 1994. https://doi.org/10.1109/86.340871

[10] N. Bu, T. Hamamoto, T. Tsuji, and O. Fukuda, "FPGA implementation of a probabilistic neural network for a bioelectric human interface," in Midwest Symposium on Circuits and Systems, 2004, vol. 3. https://doi.org/10.1109/MWSCAS.2004.1354283

[11] M. Khezri and M. Jahed, "Real-time intelligent pattern recognition algorithm for surface EMG signals," BioMedical Engineering Online, vol. 6, no. 1, p. 45, Dec. 2007. https://doi. org/10.1186/1475-925X-6-45

[12] G. Tsenov, A. H. Zeghbib, F. Palis, N. Shoylev, and V. Mladenov, "Neural networks for online classification of hand and finger movements using surface EMG signals," in 8th Seminar on Neural Network Applications in Electrical Engineering, Neurel-2006 Proceedings, 2006, pp. 167-171. https://doi.org/10.1109/NEUREL.2006.341203

[13] L. J. Hargrove, K. Englehart, and B. Hudgins, "A comparison of surface and intramuscular myoelectric signal classification," IEEE Transactions on Biomedical Engineering, vol. 54, no. 5, pp. 847-853, May 2007. https://doi.org/10.1109/TBME.2006.889192

[14] E. M. El-Daydamony, M. El-Gayar, and F. Abou-Chadi, "A computerized system for SEMG signals analysis and classification," in National Radio Science Conference, NRSC, Proceedings, 2008. https://doi.org/10.1109/NRSC.2008.4542388

[15] J. Kim, S. Mastnik, and E. André, "EMG-based hand gesture recognition for realtime biosignal interfacing," in Proceedings of the 13th international conference on Intelligent user interfaces - IUI '08, 2008, p. 30. https://doi.org/10.1145/1378773.1378778

[16] J. Chiang, Z. J. Wang, and M. J. McKeown, "A hidden Markov, multivariate autoregressive (HMM-mAR) network framework for analysis of surface EMG (sEMG) data," IEEE Transactions on Signal Processing, vol. 56, no. 8 II, pp. 4069-4081, Aug. 2008. https://doi.org/10.1109/TSP.2008.925246

[17] "12 Classification of Hand Motion Using Surface EMG Signals - Biologically Inspired Robotics [Book]." https://doi.org/10.1201/b11365-12 (accessed Mar. 11, 2020).

[18] D. Morris, T. Scott Saponas, and D. Tan, "Emerging input technologies for alwaysavailable mobile interaction," Foundations and Trends in Human-Computer Interaction, vol. 4, no. 4. pp. 245-316, 2010. https://doi.org/10.1561/1100000023

[19] M. Piyaneeranart and M. Ketcham, "Automatically Moving Robot Intended for the Elderly with Voice Control," International Journal of Online and Biomedical Engineering (iJOE), vol. 17, no. 06, pp. 19-48, Jun. 2021, Accessed: Sep. 21, 2021. https://doi.org/10.3991/ij oe.v17i06.22299

[20] M. R. Ahsan, M. I. Ibrahimy, and O. O. Khalifa, "EMG Signal Classification for Human Computer Interaction: A Review," 2009. Accessed: Nov. 30, 2019. [Online]. Available: http://www.eurojournals.com/ejsr.htm

[21] A. Chowdhury, R. Ramadas, and S. Karmakar, "Muscle Computer Interface: A Review," 2013, pp. 411-421.

[22] H. P. C. A., P. R. V. R., M. A. M. S. A., T. W. J., M. R. K., and H. M. D., "A Proposed Web Based Real Time Brain Computer Interface (BCI) System for Usability Testing," International Journal of Online and Biomedical Engineering (iJOE), vol. 15, no. 08, pp. 108-119, May 2019, Accessed: Sep. 21, 2021. https://doi.org/10.3991/ijoe.v15i08.10406 
[23] E. A. C. Advisor, "Design of a High-Resolution Surface Electromyogram (EMG) Conditioning Circuit A Major Qualifying Project Report," 2012.

[24] M. Zecca, S. Micera, M. C. Carrozza, and P. Dario, "Control of Multifunctional Prosthetic Hands by Processing the Electromyographic Signal," Critical reviews in biomedical engineering, vol. 45, no. 1-6. NLM (Medline), pp. 383-410, 2017. https://doi.org/10.1615/ critrevbiomedeng.v45.i1-6.150

[25] W. Budiharto, "ICIC Express Letters ICIC International(C2019 ISSN," vol. 13, no. 1, pp. 77-82, 2019. http://doi.org/10.24507/icicel.13.01.77

[26] S. S. Joshi, A. S. Wexler, C. Perez-Maldonado, and S. Vernon, "Brain-muscle-computer interface using a single surface electromyographic signal: Initial results," in 2011 5th International IEEE/EMBS Conference on Neural Engineering, NER 2011, 2011, pp. 342347. https://doi.org/10.1109/NER.2011.5910557

[27] M. A. Landa-Jiménez, P. González-Gaspar, C. Pérez-Estudillo, M. L. López-Meraz, C. Morgado-Valle, and L. Beltran-Parrazal, "Open-box muscle-computer interface: Introduction to human-computer interactions in bioengineering, physiology, and neuroscience courses," Advances in Physiology Education, vol. 40, no. 1, pp. 119-122, Mar. 2016. https://doi.org/10.1152/advan.00009.2015

[28] S. Vernon and S. S. Joshi, "Multidimensional control using a mobile-phone based brainmuscle-computer interface," in Proceedings of the Annual International Conference of the IEEE Engineering in Medicine and Biology Society, EMBS, 2011, pp. 5188-5194. https://doi.org/10.1109/IEMBS.2011.6091284

[29] S. Vernon and S. S. Joshi, "Brain-muscle-computer interface: Mobile-phone prototype development and testing," IEEE Transactions on Information Technology in Biomedicine, vol. 15, no. 4, pp. 531-538, Jul. 2011. https://doi.org/10.1109/TITB.2011.2153208

[30] S. Baudry, M. A. Minetto, and J. Duchateau, "Surface EMG applications in neurophysiology," in Surface Electromyography: Physiology, Engineering and Applications, Hoboken, New Jersey: Wiley-IEEE Press, 2016, pp. 333-360. https://doi.org /10.1002/9781119082934.ch12

[31] "(No Title)." https://cdn.sparkfun.com/datasheets/Sensors/Biometric/MyowareUser ManualAT-04-001.pdf (accessed Mar. 14, 2020).

[32] "Know About Different Types of Arduino Boards And Their Uses." https://www.watelectr onics.com/different-types-arduino-boards-uses/ (accessed Mar. 14, 2020).

[33] "Introduction to Servo Motors." https://www.sciencebuddies.org/science-fair-projects/refer ences/introduction-to-servo-motors (accessed Mar. 14, 2020).

\section{Author}

Hadeel K. Aljobouri received the B.S. degree in Biomedical Engineering from the University of Baghdad, Baghdad, Iraq, in 2000 the M.Sc. in Medical Engineering from Al-Nahrain University, Baghdad, Iraq, in 2004, and the Ph.D. at the Electrical and Electronics Engineering Department, Graduate School of Natural Science/ Ankara Yıldırım Beyazıt University in Turkey. She worked as an Assistant Professor at the Biomedical Engineering Department at Al-Nahrain University in Iraq. Her research interests are biomedical signal processing, medical imaging, data mining, clustering techniques, and machine learning. She has many publications in the field of biomedical engineering.

Article submitted 2021-09-21. Resubmitted 2021-11-19. Final acceptance 2021-11-21. Final version published as submitted by the author. 